\title{
Chronic obstructive pulmonary disease and the risk of cardiovascular diseases
}

\author{
Cornelia Schneider • Ulrich Bothner • \\ Susan S. Jick • Christoph R. Meier
}

Received: 20 March 2009/Accepted: 5 February 2010/Published online: 27 February 2010

(C) Springer Science+Business Media B.V. 2010

\begin{abstract}
Previous large epidemiological studies reporting on the association between chronic obstructive pulmonary disease (COPD) and cardiovascular diseases mainly focussed on prevalent diseases rather than on the incidence of newly diagnosed cardiovascular outcomes. We used the UK-based General Practice Research Database (GPRD) to assess the prevalence and incidence of cardiovascular diseases in COPD patients aged 40-79 between 1995 and 2005, and we randomly matched COPDfree comparison patients to COPD patients. In nested-case control analyses, we compared the risks of developing an incident diagnosis of cardiac arrhythmias, venous thromboembolism, myocardial infarction, or stroke between patients with and without COPD, stratifying the analyses by COPD-severity, using COPD-treatment as proxy for disease severity. We identified 35,772 patients with COPD and the same number of COPD-free patients. Most
\end{abstract}

C. Schneider - C. R. Meier

Basel Pharmacoepidemiology Unit, Division of Clinical

Pharmacy and Epidemiology, Department of Pharmaceutical

Sciences, University of Basel, Basel, Switzerland

U. Bothner

Nycomed GmbH, Constance, Germany

S. S. Jick · C. R. Meier

Boston Collaborative Drug Surveillance Program, Boston

University School of Medicine, Lexington, MA, USA

C. R. Meier $(\bowtie)$

Hospital Pharmacy, University Hospital Basel, Spitalstrasse 26, 4031 Basel, Switzerland

e-mail: meierch@uhbs.ch cardiovascular diseases were more prevalent among COPD patients than among the comparison group of COPD-free patients. The relative risk estimates of developing an incident diagnosis of cardiac arrhythmia (OR 1.19, 95\% CI 0.98-1.43), deep vein thrombosis (OR 1.35, 95\% CI $0.97-$ 1.89), pulmonary embolism (OR 2.51, 95\% CI 1.62-3.87), myocardial infarction (OR $1.40,95 \%$ CI $1.13-1.73$ ), or stroke (OR 1.13, 95\% CI 0.92-1.38), tended to be increased for patients with COPD as compared to COPDfree controls. The findings of this large observational study provide further evidence that patients with COPD are at increased risk for most cardiovascular diseases.

Keywords Arrhythmia .

Chronic obstructive pulmonary disease .

Deep vein thrombosis - Myocardial infarction .

Pulmonary embolism $\cdot$ Stroke 
PE Pulmonary embolism

Py Person years

TIA Transient ischemic attack

\section{Introduction}

Chronic obstructive pulmonary disease (COPD) is increasingly recognized as a respiratory disease with an important systemic component [1, 2]. Persistent low-level systemic inflammation is present in COPD, reflected for example by elevated levels of C-reactive protein [3]. Systemic inflammation has also been associated with atherosclerosis, ischemic heart disease and stroke [4]. Cardiovascular events are an important cause of morbidity and mortality among COPD patients [5, 6]. Apart from a possible direct association between COPD and adverse cardiovascular outcomes, COPD therapy with beta-agonists is of concern [7] due to the potential of increasing the risk for cardiac arrhythmia and myocardial infarction. In addition, COPD patients may require treatment with macrolides and quinolone antibiotics [8] which themselves may also cause adverse cardiovascular effects, and COPD and cardiovascular diseases also share smoking as an important risk factor [9].

Regardless of the underlying mechanism, various studies indicate an association between COPD and the risk of cardiovascular diseases [10-12]. According to the ACC/ AHA/ESC 2006 guidelines for the management of patients with atrial fibrillation, supraventricular arrhythmias are common in patients with COPD [13], and pulmonary embolism has frequently been observed during exacerbations of COPD $[14,15]$. Patients with COPD who experienced a myocardial infarction were at a twofold increased risk of dying within one-year after the myocardial infarction compared to patients without COPD [16], and in another study COPD patients were at an increased risk of fatal strokes when compared to COPD-free patients [17]. A Canadian study based on data from Saskatchewan assessed the period prevalence of cardiovascular events and incidence of hospitalisations and mortality from cardiovascular outcomes in COPD patients [18, 19] and a study based on the Kaiser Permanente Medical Care Program [20] provided additional information on the incidence of hospitalisations for cardiovascular outcomes.

In the current study, we quantify the risks of developing an incident diagnosis of cardiac arrhythmia, myocardial infarction (MI), deep vein thrombosis (DVT), pulmonary embolism (PE), stroke or transient ischemic attack (TIA) in relation to COPD using primary-care data from the United Kingdom (UK) between 1995 and 2005.

\section{Methods}

Data source

We used the UK-based General Practice Research Database (GPRD) to conduct a follow-up study with a nested casecontrol analysis. The GPRD has been described in detail elsewhere [21]. It is a large primary care database established in 1987 which encompasses some five million patients who are enrolled with selected general practitioners (GPs) throughout the UK. The General Practitioners (GPs) who contribute data to the GPRD have been trained to record medical information in a standard manner and to supply it anonymously. The recorded information includes demographics, medical diagnoses, and virtually all drug prescriptions. The patients enrolled in the GPRD are representative of the UK population with respect to age, sex and geographical distribution [21]. GPRD data have been previously used for studies involving COPD [22], cardiac arrhythmia [23], DVT and PE [24], MI [25], and stroke [26]. The GPRD is managed by the Medicines and Healthcare products Regulatory Agency (MHRA). The study protocol was approved by the Independent Scientific Advisory Committee (ISAC) for MHRA database research. The investigators only had access to anonymized information.

\section{Study population}

We identified in the GPRD all patients with a first-time diagnosis of COPD between January 1, 1995 and December 31, 2005 and who were aged 40-79 years. We excluded patients with less than 3 years of active recording history prior to the first COPD diagnosis. In addition, we identified at random from the GPRD one COPD-free comparison subject for each COPD patient, matched 1:1 on age (same year of birth), sex, general practice and calendar time (i.e. on the date of the first COPD diagnosis of the COPD patient). We applied the same exclusion criteria to the comparison group as to the COPD patients. Within this study population we assessed and compared the prevalence of diagnosed cardiovascular diseases prior to the COPD diagnosis or the corresponding date in the comparison group. We then excluded all patients from the COPD group and from the comparison group who had a recorded history of congestive heart failure, MI, DVT, pulmonary embolism (PE), stroke, TIA, cardiac arrhythmia, cancer (except non-melanoma skin cancer), HIV, drug abuse, or alcoholism prior to the first COPD diagnosis (or the corresponding date in the COPD-free group).

Follow-up analysis

We followed all remaining patients from the start of follow-up (i.e. the COPD diagnosis date or the corresponding 
date in the comparison group) and assessed the person time for each patient until he or she developed an incident diagnosis of arrhythmia, DVT, PE, MI, stroke/TIA, left the database, died or reached the end of the study (December 31, 2005), whichever came first. As we excluded patients with prevalent cardiovascular diseases prior to the start date both from the COPD and the COPD-free comparison group, the two groups were no longer of equal size for the follow-up analysis. We followed each patient up to the first cardiovascular outcome; if more than one outcome was recorded during follow-up, the patient was only followed up to the first endpoint. We estimated incidence rates with $95 \%$ confidence intervals (CI) separately for each outcome.

\section{Nested case-control analysis}

We conducted nested case-control analyses to further analyze the impact of COPD severity and various potential confounders on the risk of developing an outcome of interest, i.e. arrhythmia, DVT, PE, MI or stroke/TIA. For this purpose we identified at random for each case with a study outcome up to 4 control patients from the study population (i.e. patients with or without COPD) who did not develop arrhythmia, DVT, PE, MI or stroke/TIA during follow-up. These controls were matched to cases on age, sex, practice and index date, i.e. the date when the case had the incident diagnosis of a cardiovascular outcome of interest. We compared the prevalence of COPD in patients with a cardiovascular endpoint and his or her controls using conditional logistic regression analyses. As a surrogate marker for COPD disease severity we used COPD treatment, similar to a previous approach published by Soriano et al. [27]. We therefore categorized COPD patients into three groups; patients with 'no treatment' (as surrogate for mild COPD), with 'drug treatment' (as surrogate for moderate COPD), defined as having had at least one prescription for short acting anticholinergics, short acting beta agonists, tiotropium, long acting beta agonists, leukotriene receptor antagonists, inhaled steroids, and/or xanthines, or with 'oxygen treatment' (as surrogate for severe COPD). We adjusted the analyses for smoking status (none, current, past, unknown), body mass index (BMI; <18.5, 18.5-24.9, 25-29.9, 30-60 kg/m ${ }^{2}$, or unknown), and for a range of comorbidities and drugs. Arrhythmia analyses are adjusted for smoking status, hypertension, use of beta agonists, xanthines, quinolones, macrolides, vitamin $\mathrm{K}$ antagonists, beta blockers, calcium channel blockers, diuretics, cardiac glycosides and coronary dilatators; PE and DVT analyses are adjusted for smoking status, BMI, hypertension and NSAID use; MI analyses are adjusted for smoking status, BMI, hypertension, hyperlipidemia, diabetes and NSAID use; Stroke/TIA analyses are adjusted for smoking status, BMI, hypertension, diabetes and use of aspirin.
Statistical analyses were performed with the statistical software SAS (release 9.1, SAS Institute, Inc., Cary, NC, USA)

\section{Case validation}

All patients with a recorded first-time diagnosis of cardiac arrhythmia, PE, DVT, MI or stroke/TIA were identified using specific disease codes recorded by the GP. We validated potential cases according to the pharmacological treatment recorded after the cardiovascular diagnosis. In order to be eligible, cases with DVT or PE had to have been hospitalised within 30 days after the diagnosis or had to die within 30 days after the diagnosis, and/or had to start treatment with heparin, vitamin $\mathrm{K}$ antagonists, platelet aggregation inhibitors, direct thrombin inhibitors or fibrinolytic enzymes within 90 (DVT) or 180 (PE) days after the diagnosis. If potential cases had one or more prescriptions for heparin, vitamin $\mathrm{K}$ antagonists, platelet aggregation inhibitors, direct thrombin inhibitors or fibrinolytic enzymes recorded more than 90 days prior to the DVT diagnosis, the potential case was excluded from the analysis. Patients with an incident MI diagnosis had to have been hospitalised within 30 days of the MI diagnosis, or had to die within 30 days after the diagnosis, and/or they had to start a new treatment with ACE antagonists, beta blockers, statins, vitamin $\mathrm{K}$ antagonists, platelet aggregation inhibitors, or aspirin within 90 days after the diagnosis. Patients with heart surgery or with prescriptions for heparin, vitamin $\mathrm{K}$ antagonists, platelet aggregation inhibitors, direct thrombin inhibitors or fibrinolytic enzymes more than 30 days prior to the diagnosis date were excluded. Stroke or TIA patients had to be hospitalised within 30 days of the stroke diagnosis, had to die within 30 days after the diagnosis, and/or had to have new treatment with aspirin, heparin, vitamin $\mathrm{K}$ antagonists, platelet aggregation inhibitors, direct thrombin inhibitors or fibrinolytic enzymes within 180 days after the diagnosis.

\section{Results}

We identified a total of 35,772 patients with a first-time COPD diagnosis between 1995 and 2005, and the same number of matched COPD-free patients in the comparison group. The total study population of 71,544 patients encompassed slightly more men $(51.3 \%)$ than women $(48.7 \%)$, and $73.3 \%$ of the study population were 60 years of age or older at the time of the first recording of a COPD diagnosis. With the exception of hypertension, most other cardiovascular diseases were more prevalent among COPD patients than among patients in the comparison group prior to the time of the first COPD diagnosis (or the 
corresponding date in the COPD-free comparison group). Characteristics of the study population are shown in Table 1.

After excluding patients with prevalent cardiovascular diseases, cancer, HIV, alcoholism or drug abuse, we identified among the remaining study population 1,191 cases with an incident cardiac arrhythmia diagnosis, 136 cases with incident pulmonary embolism, 210 cases with incident DVT, 511 cases with incident MI, and 598 cases with an incident stroke/TIA diagnosis. Incidence rates of all cardiovascular endpoints were higher among COPD patients than among patients without COPD; the incidence rate of $\mathrm{PE}$ was more than twice as high $(12.8 / 10,000$ py among COPD patients vs. 5.4/10,000 py in the comparison group). In absolute terms, the incidence rates were highest for arrhythmia (91.1/10,000 py among COPD patients and $66.7 / 10,000$ py in the comparison group), and lowest for

Table 1 Characteristics of the study population

\begin{tabular}{|c|c|c|c|c|c|}
\hline & \multicolumn{2}{|l|}{ COPD } & \multicolumn{2}{|c|}{ COPD Free } & \multirow[t]{2}{*}{ OR $(95 \% \mathrm{CI})^{*}$} \\
\hline & \# & $\%$ & \# & $\%$ & \\
\hline Male & 18,361 & 51.3 & 18,361 & 51.3 & - \\
\hline \multicolumn{6}{|c|}{ Age groups (years) } \\
\hline $40-49$ & 2,432 & 6.8 & 2,431 & 6.8 & - \\
\hline $50-59$ & 7,101 & 19.9 & 7,105 & 19.9 & - \\
\hline $60-69$ & 12,075 & 33.8 & 12,072 & 33.8 & - \\
\hline$>70$ & 14,164 & 39.6 & 14,164 & 39.6 & - \\
\hline \multicolumn{6}{|l|}{ Smoking status } \\
\hline Non smoker & 7,722 & 21.6 & 18,030 & 50.4 & $1.00 \quad$ (ref) \\
\hline Current smoker & 15,472 & 43.3 & 6,428 & 18.0 & $6.27(5.99-6.57)$ \\
\hline Ex-smoker & 9,054 & 25.3 & 6,431 & 18.0 & $3.65(3.48-3.83)$ \\
\hline Unknown & 3,524 & 9.8 & 4,883 & 13.7 & $1.50(1.41-1.60)$ \\
\hline \multicolumn{6}{|l|}{ BMI $\left(\mathrm{kg} / \mathrm{m}^{2}\right)$} \\
\hline $15.0-18.4$ & 1,301 & 3.6 & 325 & 0.9 & $3.56(3.14-4.03)$ \\
\hline $18.5-24.9$ & 12,026 & 33.6 & 10,678 & 29.9 & 1.00 (ref) \\
\hline $25.0-29.9$ & 9,643 & 27.0 & 11,647 & 32.6 & $0.74(0.71-0.76)$ \\
\hline $30.0-60.0$ & 5,660 & 15.8 & 5,690 & 15.9 & $0.89(0.85-0.93)$ \\
\hline Unknown & 7,142 & 20.0 & 7,432 & 20.8 & $0.84(0.80-0.88)$ \\
\hline Arrhythmia & 2,589 & 7.2 & 2,291 & 6.4 & $1.15(1.08-1.21)$ \\
\hline $\mathrm{CHF}$ & 2,991 & 8.4 & 1,253 & 3.5 & $2.62(2.44-2.81)$ \\
\hline DVT & 790 & 2.2 & 715 & 2.0 & $1.11(1.00-1.23)$ \\
\hline Hypertension & 9,782 & 27.4 & 11,353 & 31.7 & $0.80(0.77-0.82)$ \\
\hline MI/IHD & 6,528 & 18.3 & 5,561 & 15.6 & $1.23(1.18-1.28)$ \\
\hline $\mathrm{PE}$ & 527 & 1.5 & 333 & 0.9 & $1.59(1.39-1.83)$ \\
\hline Stroke/TIA & 2,454 & 6.9 & 2,093 & 5.9 & $1.19(1.12-1.26)$ \\
\hline
\end{tabular}

$B M I$ body mass index, ref reference group, $C H F$ congestive heart failure, $C I$ confidence interval, $I H D$ ischemic heart disease, $M I$ myocardial infarction, $O R$ odds ratio, $P E$ pulmonary embolism, ref reference, TIA transient ischemic attack. For all cardiovascular diseases each disease-free status is the referent

* OR from univariate analyses, but adjusted for age, sex, GP and index date by matching
PE. The various incidence rates, stratified by gender, are displayed in detail in Table 2.

In order to evaluate the effect of COPD severity on the risk of developing a study outcome of interest, we conducted a nested case-control analysis, where we stratified COPD patients according to their treatment pattern into mild, moderate or severe COPD. The relative risk of developing arrhythmia overall was similar for patients with or without COPD (OR 1.19, 95\% CI 0.98-1.43), and COPD severity did not alter the risk estimate substantially.

Table 2 Incidence rates of cardiovascular diseases in COPD and control patients

\begin{tabular}{|c|c|c|c|c|}
\hline & \# Cases & $\begin{array}{l}\text { Person } \\
\text { years (py) }\end{array}$ & $\begin{array}{l}\text { IR per } \\
10,000 \text { py }\end{array}$ & $95 \% \mathrm{CI}$ \\
\hline \multicolumn{5}{|l|}{ Arrhythmia } \\
\hline COPD-Free & 563 & 84435.2 & 66.7 & $(61.4-72.4)$ \\
\hline Men & 288 & 39532.0 & 72.9 & $(64.9-81.7)$ \\
\hline Women & 275 & 44903.3 & 61.2 & $(54.4-68.9)$ \\
\hline COPD & 628 & 68958.5 & 91.1 & $(84.3-98.4)$ \\
\hline Men & 334 & 32432.2 & 103.0 & $(92.6-114.6)$ \\
\hline Women & 294 & 36526.2 & 80.5 & $(71.8-90.2)$ \\
\hline \multicolumn{5}{|l|}{$\mathrm{PE}$} \\
\hline COPD-Free & 46 & 85765.1 & 5.4 & $(4.0-7.2)$ \\
\hline Men & 23 & 40260.6 & 5.7 & $(3.8-8.6)$ \\
\hline Women & 23 & 45504.4 & 5.1 & $(3.4-7.6)$ \\
\hline COPD & 90 & 70233.0 & 12.8 & $(10.4-15.8)$ \\
\hline Men & 48 & 33258.3 & 14.4 & $(10.9-19.1)$ \\
\hline Women & 42 & 36974.8 & 11.4 & $(8.4-15.4)$ \\
\hline \multicolumn{5}{|l|}{ DVT } \\
\hline Controls & 96 & 85765.1 & 11.2 & $(9.2-13.7)$ \\
\hline Men & 39 & 40260.6 & 9.7 & $(7.1-13.2)$ \\
\hline Women & 57 & 45504.4 & 12.5 & $(9.7-16.2)$ \\
\hline COPD & 114 & 70233.0 & 16.2 & $(13.5-19.5)$ \\
\hline Men & 53 & 33258.3 & 15.9 & $(12.2-20.8)$ \\
\hline Women & 61 & 369774.8 & 16.5 & $(12.9-21.2)$ \\
\hline \multicolumn{5}{|l|}{ MI } \\
\hline COPD-Free & 224 & 85538.9 & 26.2 & $(23.0-29.8)$ \\
\hline Men & 147 & 39990.9 & 36.8 & $(31.3-43.2)$ \\
\hline Women & 77 & 45548.0 & 16.9 & $(13.5-21.1)$ \\
\hline COPD & 287 & 70055.2 & 41.0 & $(36.5-46.0)$ \\
\hline Men & 174 & 33000.8 & 52.7 & $(45.5-61.1)$ \\
\hline Women & 113 & 37054.3 & 30.5 & $(25.4-36.7)$ \\
\hline \multicolumn{5}{|l|}{ Stroke/TIA } \\
\hline COPD-Free & 298 & 84586.7 & 35.2 & $(31.5-39.5)$ \\
\hline Men & 165 & 39554.0 & 41.7 & $(35.8-48.6)$ \\
\hline Women & 133 & 45032.7 & 29.5 & $(24.9-35.0)$ \\
\hline COPD & 300 & 69510.2 & 43.2 & $(38.6-48.3)$ \\
\hline Men & 134 & 32857.9 & 40.8 & $(34.5-48.3)$ \\
\hline Women & 166 & 36652.2 & 45.3 & $(38.9-52.7)$ \\
\hline
\end{tabular}

$I R$ incidence rate, $C I$ confidence interval 
The same is true for the association between COPD and the risk of stroke/TIA with an overall relative risk estimate of 1.13 (95\% CI 0.92-1.38) for COPD patients as compared to patients without COPD. For MI, the relative risk estimate was highest for patients with severe COPD (OR 3.00, 95\% CI 1.53-5.86), while the relative risk of PE was increased across all categories of COPD severity with a particularly high risk for patients with severe COPD, based on only 8 cases and 6 controls (OR 7.47, 95\% CI 2.3523.7). Overall, the risk of DVT was 1.35 (95\% CI $0.97-$ 1.89) for COPD patients compared to COPD-free controls. The findings for the various cardiovascular outcomes, stratified by COPD severity, are displayed in Table 3 .

In addition to a range of potential confounders, we also adjusted the model on arrhythmias for use of potentially arrhythmic drugs (e.g. certain antibiotics) prior to the index date. In order to test whether use of such drugs may also play a role in the risk of developing other cardiovascular outcomes, we also adjusted the analyses on all other cardiovascular outcomes for use of potentially arrhythmic drugs, but this did not alter the findings materially (data not shown).

\section{Discussion}

In this large observational study, we explored the association between COPD and the risk of developing an incident diagnosis of cardiac arrhythmia, DVT, PE, MI, or stroke/ TIA. We quantified the prevalence of these diseases prior to the first COPD diagnosis, and we compared their incidence rates between COPD patients and a matched comparison group free of COPD. In general, the findings for the association between cardiovascular diseases and COPD in our study are comparable to results from studies in other health care settings and/or other countries.
Table 3 Case-Control analysis of the risk of various cardiovascular diseases stratified by COPD severity
$O R$ odds ratio, ref reference value, $C I$ confidence interval, \# number of

${ }^{a}$ Arrhythmia analyses are adjusted for smoking status, hypertension, beta agonist use, xanthine use, quinolone use, macrolide use, vitamin $\mathrm{K}$ antagonist use and use of beta blockers, calcium channel blockers, diuretics, cardiac glycosides and coronary dilatators; PE and DVT analyses are adjusted for smoking status, BMI, hypertension and NSAID use; MI analyses are adjusted for smoking status, BMI, hypertension, hyperlipidemia, diabetes and NSAID use; Stroke/TIA analyses are adjusted for smoking status, BMI, hypertension, aspirin use and diabetes

\begin{tabular}{|c|c|c|c|c|}
\hline & \# Cases & \# Controls & Crude OR $(95 \% \mathrm{CI})$ & Adjusted $^{\mathrm{a}}$ OR $(95 \% \mathrm{CI})$ \\
\hline \multicolumn{5}{|l|}{ Arrhythmia } \\
\hline No COPD & 563 & 3,116 & 1.00 (ref) & 1.00 (ref) \\
\hline Any COPD & 628 & 2,418 & $1.42(1.25-1.61)$ & $1.19(0.98-1.43)$ \\
\hline Mild & 48 & 191 & $1.42(1.01-2.00)$ & $1.64(1.14-2.34)$ \\
\hline Moderate & 548 & 2,146 & $1.39(1.22-1.59)$ & $1.07(0.86-1.32)$ \\
\hline Severe & 32 & 81 & $2.10(1.36-3.23)$ & $1.29(0.79-2.11)$ \\
\hline \multicolumn{5}{|l|}{ PE } \\
\hline No COPD & 46 & 314 & 1.00 (ref) & 1.00 (ref) \\
\hline Any COPD & 90 & 230 & $2.57(1.74-3.78)$ & $2.51(1.62-3.87)$ \\
\hline Mild & 7 & 18 & $2.61(1.03-6.64)$ & $3.58(1.32-9.70)$ \\
\hline Moderate & 75 & 206 & $2.39(1.60-3.58)$ & $2.23(1.42-3.50)$ \\
\hline Severe & 8 & 6 & $8.58(2.88-25.6)$ & $7.47(2.35-23.7)$ \\
\hline \multicolumn{5}{|l|}{ DVT } \\
\hline No COPD & 96 & 454 & 1.00 (ref) & 1.00 (ref) \\
\hline Any COPD & 114 & 386 & $1.38(1.02-1.86)$ & $1.35(0.97-1.89)$ \\
\hline Mild & 10 & 26 & $1.78(0.84-3.77)$ & $1.73(0.78-3.80)$ \\
\hline Moderate & 100 & 334 & $1.41(1.03-1.91)$ & $1.37(0.97-1.94)$ \\
\hline Severe & 4 & 26 & $0.70(0.24-2.06)$ & $0.79(0.26-2.39)$ \\
\hline \multicolumn{5}{|l|}{ MI } \\
\hline No COPD & 224 & 1,082 & 1.00 (ref) & 1.00 (ref) \\
\hline Any COPD & 287 & 813 & $1.70(1.39-2.06)$ & $1.40(1.13-1.73)$ \\
\hline Mild & 33 & 64 & $2.43(1.56-3.81)$ & $1.79(1.12-2.86)$ \\
\hline Moderate & 238 & 726 & $1.58(1.29-1.94)$ & $1.30(1.04-1.62)$ \\
\hline Severe & 16 & 23 & $3.11(1.62-5.95)$ & $3.00(1.53-5.86)$ \\
\hline \multicolumn{5}{|l|}{ Stroke/TIA } \\
\hline No COPD & 298 & 1,256 & 1.00 (ref) & 1.00 (ref) \\
\hline Any COPD & 300 & 1,004 & $1.25(1.05-1.49)$ & $1.13(0.92-1.38)$ \\
\hline Mild & 20 & 66 & $1.28(0.77-2.15)$ & $1.22(0.71-2.09)$ \\
\hline Moderate & 271 & 900 & $1.26(1.05-1.51)$ & $1.13(0.92-1.38)$ \\
\hline Severe & 9 & 38 & $0.98(0.47-2.05)$ & $1.00(0.47-2.15)$ \\
\hline
\end{tabular}


$\mathrm{PE}$ is a relatively rare outcome, but the association between COPD and PE was rather strong (OR 2.51, 95\% CI 1.62-3.87) with incidence rates increased both in men and women with COPD compared to COPD-free controls. It is difficult to tell whether this association is a causal one or possibly the result of some diagnostic bias, since PE has been reported to be frequently observed during exacerbations of COPD [14, 15]. The authors of a French prospective cohort study investigated PE in COPD patients with unexplained exacerbations and reported a prevalence of PE of $25 \%$ [14]. Sidney et al. [20] reported an age-adjusted case patient rate per 100,000 py for hospitalized PE of 129.4 among COPD patients and a 2.74 (95\% CI 1.99-3.76)-fold increased relative risk of hospitalisation due to $\mathrm{PE}$ among COPD patients compared to control patients, adjusted for age, gender and cardiovascular risk morbidities. The RR in women of 2.32 (95\% CI 1.58-3.41) is closely similar to the crude IRR 2.25 (1.38-3.66) which we found, however, their RR in men was slightly higher than in our analysis. A study based on Saskatchewan data reported a 5.46 (95\% CI 4.257.02)-fold increased relative risk of PE for COPD patients when compared to control patients [18]. DVT, often preceding pulmonary embolism, was not associated with a substantially altered relative COPD risk when compared to the COPD-free comparison group in our study.

In absolute terms, cardiac arrhythmia was the most frequent cardiovascular outcome observed in our study population. COPD patients-both men and women-were slightly more likely to have an incident arrhythmia diagnosis recorded than patients without COPD. Part of this association might be explained by a higher exposure to arrhythmogenic drugs such as beta agonists, a mainstay in COPD therapy [7], and quinolone or macrolide antibiotics for bacterial infections [8]. Hypoxemia and hypercapnia in COPD patients may also contribute to an increased risk of cardiac arrhythmias as they increase the QTc dispersion (QTcD) [28]. The authors of a Danish study focussed on atrial fibrillation among COPD patients and observed an increased risk associated with reduced lung function during a five-year follow-up [29]. Previously reported baseline prevalences of arrhythmia in COPD vary; Sidney et al. [20] reported that $4.7 \%$ of COPD patients had atrial fibrillation and $2.7 \%$ other arrhythmias, while a study in American Veterans found a prevalence of arrhythmias of $14.2 \%$ [30]. A comparison of the prevalence of arrhythmias in a Canadian study found an OR of 1.76 (95\% CI 1.64-1.89) when comparing COPD patients with COPD-free patients. This study did not exclude patients with a history of cardiovascular events, but the authors adjusted the analysis for cardiovascular comorbidities [18]. The authors of another observational study stratified by COPD severity and reported relative risk estimates associated with various levels of COPD severity ranging from 0.92 (mild COPD) to 1.27 (severe COPD) [17].
We observed a rather strong association between severe COPD and the risk of developing an incident MI (OR 3.00, 95\% CI 1.53-5.86], after adjusting for smoking, a common risk factor of COPD and MI. Persistent low-level systemic inflammation, reflected by increased levels of the C-reactive protein (CRP), is often present in patients with COPD [3], and increased levels of CRP are associated with an increased MI risk. Several previous observational studies have reported an association between COPD and MI [18, 20], while Engström et al. [31] did not detect an association between low $\mathrm{FEV}_{1}$ and cardiac events, defined as fatal or non-fatal MI or death from chronic ischemic heart disease. The association between COPD and risk of MI was higher in women than in men, an observation which was also reported by Sydney et al. [20].

We found only a weak association between COPD and the risk of stroke/TIA with an OR of 1.13 (95\% CI $0.92-$ 1.38). While the crude incidence rates in men with or without COPD were closely similar, COPD was associated with a 1.5 -fold increased risk in women. Sidney et al. also reported a RR of 1.50 (95\% CI 1.30-3.41) in women with COPD. [20] Truelsen et al. [32] explored the association between reduced lung function and the risk of stroke, resulting in ORs ranging between 1.00 and 1.38 for $\mathrm{FEV}_{1}$ values below $80 \%$ when compared to $\mathrm{FEV}_{1}$ values $\geq 100 \%$. Hozawa et al. [33] did not find a significant association between airway obstruction and ischemic stroke. Engström et al. [31] did not observe a statistically significantly altered risk when they studied the association between low $\mathrm{FEV}_{1}$ and stroke. Two other longitudinal studies found only weak associations between COPD and stroke $[18,20]$. Thus, COPD does not seem to substantially alter the risk of developing a first-time diagnosis of stroke. Whether the slightly different findings for MI and stroke/ TIA risks in association with COPD indeed reflect a greater vulnerability of women, or whether this is due to residual confounding e.g. by smoking needs further investigation.

Several limitations of our study need to be addressed. As this study is based on data from the primary-care setting, there is a possibility that we might have missed some cases with COPD, especially patients with milder forms of COPD who have not yet been diagnosed. Thus, our results only apply to GP-diagnosed COPD diagnoses, and the same holds true for the cardiovascular outcomes. However, as most of the cardiovascular outcomes in this study represent acute and rather severe diseases, we can assume that few cases with an outcome of interest would have been missed. We decided a priori to analyze only the first cardiovascular event during follow-up; the various cardiovascular outcomes are highly related to each other and their treatment and management is similar, thus a first episode of a cardiovascular outcome materially alters the risk of developing a second. It would have been desirable to have 
(more) information on potential risk factors for the outcomes such as immobility, disease severity or socioeconomic status. We assessed COPD severity using medication as a proxy in the nested case-control analysis, and we stratified the analyses by this parameter. However, we were not able to classify the large group of patients with moderate COPD (i.e. those with some pharmacological treatment) in more detail with these data. By matching cases and controls on practice, we controlled to some degree for socioeconomic status, as social deprivation shows a geographical pattern and therefore people from the same area are more likely to see the same GP. On the other hand, it is a strength of the this high-quality data source that it encompassed a large population with a considerably long follow-up of up to 10 years, and this database has already proven to be of high validity for studies of COPD and various cardiovascular outcomes. Another advantage of the GPRD, for example compared with other settings such as the study from the Kaiser Permanente Medical Care Program, is the availability of information on lifestyle factors, in this case particularly on smoking. Collecting information from different settings is important as the pattern and the impact of factors, such as smoking or health care system, may vary.

Acknowledgments Source of funding: This study was funded by an unconditional grant by Nycomed GmbH. Financial Disclosure: Dr. Ulrich Bothner was employed by Nycomed $\mathrm{GmbH}$ at that time.

\section{References}

1. Agusti A, Soriano JB. COPD as a systemic disease. Copd. 2008;5(2):133-8.

2. GOLD. Executive Summary: Global Strategy for the Diagnosis, Management, and Prevention of COPD. 2007 http://www.gold copd.org/Guidelineitem.asp?11=2\&12=1\&intId=996.

3. Pinto-Plata VM, Mullerova H, Toso JF, Feudjo-Tepie M, Soriano JB, Vessey RS, et al. C-reactive protein in patients with COPD, control smokers and non-smokers. Thorax. 2006;61(1):23-8.

4. Ridker PM. Clinical application of C-reactive protein for cardiovascular disease detection and prevention. Circulation. 2003; 107(3):363-9.

5. Huiart L, Ernst P, Suissa S. Cardiovascular morbidity and mortality in COPD. Chest. 2005;128(4):2640-6.

6. Hansell AL, Walk JA, Soriano JB. What do chronic obstructive pulmonary disease patients die from? a multiple cause coding analysis. Eur Respir J. 2003;22(5):809-14.

7. Salpeter SR, Ormiston TM, Salpeter EE. Cardiovascular effects of beta-agonists in patients with asthma and COPD: a metaanalysis. Chest. 2004;125(6):2309-21.

8. Owens RC Jr, Nolin TD. Antimicrobial-associated QT interval prolongation: pointes of interest. Clin Infect Dis. 2006;43(12): 1603-11.

9. Giovino GA. The tobacco epidemic in the United States. Am J Prev Med. 2007;33(6 Suppl):S318-26.

10. Sin DD, Man SF. Chronic obstructive pulmonary disease: a novel risk factor for cardiovascular disease. Can J Physiol Pharmacol. 2005;83(1):8-13.

11. Johnston AK, Mannino DM, Hagan GW, Davis KJ, Kiri VA. Relationship between lung function impairment and incidence or recurrence of cardiovascular events in a middle-aged cohort. Thorax. 2008;63(7):599-605.

12. Rodriguez-Roisin R, Soriano JB. Chronic obstructive pulmonary disease with lung cancer and/or cardiovascular disease. Proceedings of the American Thoracic Society. 2008;5(8):842-7.

13. Fuster V, Ryden LE, Cannom DS, Crijns HJ, Curtis AB, Ellenbogen KA, et al. ACC/AHA/ESC 2006 guidelines for the management of patients with atrial fibrillation: full text: a report of the American College of Cardiology/American Heart Association Task Force on practice guidelines and the European Society of Cardiology Committee for Practice Guidelines (Writing Committee to Revise the 2001 guidelines for the management of patients with atrial fibrillation) developed in collaboration with the European Heart Rhythm Association and the Heart Rhythm Society. Europace. 2006;8(9):651-745.

14. Tillie-Leblond I, Marquette CH, Perez T, Scherpereel A, Zanetti $\mathrm{C}$, Tonnel $\mathrm{AB}$, et al. Pulmonary embolism in patients with unexplained exacerbation of chronic obstructive pulmonary disease: prevalence and risk factors. Ann Intern Med. 2006;144(6): 390-6.

15. Erelel M, Cuhadaroglu C, Ece T, Arseven O. The frequency of deep venous thrombosis and pulmonary embolus in acute exacerbation of chronic obstructive pulmonary disease. Respir Med. 2002;96(7):515-8.

16. Salisbury AC, Reid KJ, Spertus JA. Impact of chronic obstructive pulmonary disease on post-myocardial infarction outcomes. Am J Cardiol. 2007;99(5):636-41.

17. Hole DJ, Watt GC, Davey-Smith G, Hart CL, Gillis CR, Hawthorne VM. Impaired lung function and mortality risk in men and women: findings from the Renfrew and Paisley prospective population study. BMJ 1996; 313(7059): 711-5; discussion 15-6.

18. Curkendall SM, DeLuise C, Jones JK, Lanes S, Stang MR, Goehring E Jr, et al. Cardiovascular disease in patients with chronic obstructive pulmonary disease, Saskatchewan Canada cardiovascular disease in COPD patients. Ann Epidemiol. 2006;16(1):63-70.

19. Curkendall SM, Lanes S, de Luise C, Stang MR, Jones JK, She D, et al. Chronic obstructive pulmonary disease severity and cardiovascular outcomes. Eur J Epidemiol. 2006;21(11):803-13.

20. Sidney S, Sorel M, Quesenberry CP Jr, DeLuise C, Lanes S, Eisner MD. COPD and incident cardiovascular disease hospitalizations and mortality: Kaiser Permanente Medical Care Program. Chest. 2005;128(4):2068-75.

21. Wood L, Martinez C. The general practice research database: role in pharmacovigilance. Drug Saf. 2004;27(12):871-81.

22. Soriano JB, Maier WC, Egger P, Visick G, Thakrar B, Sykes J, et al. Recent trends in physician diagnosed COPD in women and men in the UK. Thorax. 2000;55(9):789-94.

23. Ruigomez A, Johansson S, Wallander MA, Garcia Rodriguez LA. Predictors and prognosis of paroxysmal atrial fibrillation in general practice in the UK. BMC Cardiovasc Disord. 2005;5:20.

24. Jick H, Jick SS, Gurewich V, Myers MW, Vasilakis C. Risk of idiopathic cardiovascular death and nonfatal venous thromboembolism in women using oral contraceptives with differing progestagen components. Lancet. 1995;346(8990):1589-93.

25. Schlienger RG, Jick H, Meier CR. Use of nonsteroidal antiinflammatory drugs and the risk of first-time acute myocardial infarction. Br J Clin Pharmacol. 2002;54(3):327-32.

26. Soedamah-Muthu SS, Fuller JH, Mulnier HE, Raleigh VS, Lawrenson RA, Colhoun HM. High risk of cardiovascular disease in patients with type 1 diabetes in the UK: a cohort study using the general practice research database. Diabetes Care. 2006; 29(4):798-804.

27. Soriano JB, Maier WC, Visick G, Pride NB. Validation of general practitioner-diagnosed COPD in the UK General practice research database. Eur J Epidemiol. 2001;17(12):1075-80. 
28. Sarubbi B, Esposito V, Ducceschi V, Meoli I, Grella E, Santangelo L, et al. Effect of blood gas derangement on QTc dispersion in severe chronic obstructive pulmonary disease: evidence of an electropathy? Int J Cardiol. 1997;58(3):287-92.

29. Buch P, Friberg J, Scharling H, Lange P, Prescott E. Reduced lung function and risk of atrial fibrillation in the Copenhagen City Heart Study. Eur Respir J. 2003;21(6):1012-6.

30. Mapel DW, Dedrick D, Davis K. Trends and cardiovascular comorbidities of COPD patients in the Veterans Administration Medical System, 1991-1999. Copd. 2005;2(1):35-41.
31. Engstrom G, Hedblad B, Valind S, Janzon L. Increased incidence of myocardial infarction and stroke in hypertensive men with reduced lung function. J Hypertens. 2001;19(2):295-301.

32. Truelsen T, Prescott E, Lange P, Schnohr P, Boysen G. Lung function and risk of fatal and non-fatal stroke. The Copenhagen City Heart Study. Int J Epidemiol. 2001;30(1):145-51.

33. Hozawa A, Billings JL, Shahar E, Ohira T, Rosamond WD, Folsom AR. Lung function and ischemic stroke incidence: the Atherosclerosis Risk in Communities study. Chest. 2006;130(6): 1642-9. 